3 Stein W. Astrophys J. 148, 689 (1967)

4 Colvin, J. D. Astrophys J. 190,515(1974)

5 Charugin, V. M. Astrophys. Space Sci. 37, 449 (1975)

6 Singh, R.P. Planet Space Sci. 20, 2073 (1972).

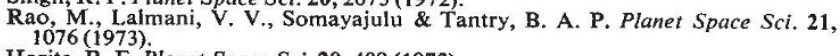

8 Horita, R. E. Planet Space Sci. 20, 409 (1972)

9 Bell, T. F. \& Buneman, D. Phys. Rev. 133A, 1300 (1964)

10 Kulsrud, R. \& Pearce, W. Astrophys. J. 156, 445 (1969).

\section{Measuring solar wind velocity with spacecraft phase scintillations}

THE discovery of interplanetary scintillations (IPS) of natural radio sources ${ }^{1}$ has been followed by extensive multiple-station or spaced-receiver IPS measurements of the solar wind velocity in regions not yet probed by direct spacecraft ${ }^{2-8}$. Few measurements have, however, been obtained within $40 R_{\odot}$ because amplitude or intensity scintillations saturate when they are strong as is the case near the Sun. This is unfortunate because the scarcity of wind velocity measurements in the acceleration region close to the Sun is one of the reasons why the construction of a unique picture of solar wind dynamics has not yet been possible, despite numerous theoretical studies and direct spacecraft observations farther out ${ }^{8,10}$. The demonstration of the measurement of spacecraft phase scintillations with a coherent dual-frequency radio system ${ }^{11}$ paves the way for making solar wind velocity measurements with multiplestation phase scintillations. Although similar in principle to the IPS technique, several factors make this new tool a more powerful and promising one.

Because phase scintillations do not saturate when they are strong, observations can be carried out closer to the Sun than with amplitude scintillations. Spectral broadening has this same property ${ }^{12}$ and we have obtained measurements at $2.3 \mathrm{GHz}$ as close as $1.7 R_{\odot}$ with the Helios spacecraft. Extensive phase scintillation observations in the accleration region of the solar wind are, therefore, possible using a dual-frequency $\mathrm{S}$ - and $\mathrm{X}$-band coherent radio link ${ }^{11}$.

For multiple-station observations the baselines are restricted to distances shorter than the correlation distance of the observed scintillations. For amplitude scintillations, the correlation distance is of the order of the size of the first Fresnel zone ${ }^{13}$, which is roughly $140 \mathrm{~km}$ at $2.3 \mathrm{GHz}$. On the other hand, since the correlation distance for phase scintillations is at least $10^{6} \mathrm{~km}$ (refs 11,13), a much wider range of baselines is possible. Particularly suited for these measurements are the three $64-\mathrm{m}$ S- and X-band tracking antennae of NASA's Deep Space Net located at Goldstone (California), Canberra and Madrid. These antennae can be used for two-station observations with baselines of the order of $10^{4} \mathrm{~km}$.

Because the slowly varying phase scintillations are considerably stronger than the faster varying amplitude scintillations ${ }^{13}$, the signal-noise ratio (SNR) needed for long baseline phase measurements is much lower than that needed for short baseline amplitude measurements. The frequency spectrum of the phase scintillations ${ }^{11,13}$ indiates that if the baseline distance is increased by a factor of 10 the SNR can be reduced by at least $20 \mathrm{~dB}$. The choice of observing antennae for phase scintillations is, therefore, flexible, since a wide range of antenna sizes and receiver noise temperatures can be considered, in addition to the wide range of baseline distances. This is especially important since it would be desirable to add observing antennae to the group of NASA 64-m antennae to permit three-station measurements. Also, for a given SNR, slowly varying phase scintillations yield measurements at larger solar elongations than faster amplitude scintillations.

We have discussed some of the important advantages of measuring solar wind velocity with multiple-station phase scintillations. Current and future NASA deep space missions equipped with the coherent $S / X$ radio system include Viking, Pioneer Venus and Mariner Jupiter/Saturn. Extensive observations using this new technique during the numerous superior conjunctions of these spacecraft will provide much needed velocity measurements in the acceleration region of the solar wind. These proposed observations will complement the large number of measurements that have been and are still being made with natural radio sources and will lead to a better understanding of the dynamics and driving mechanisms of the solar wind.

This work was supported by NASA.

Jet Propulsion Laboratory,

RICHARD WOO

California Institute of Technology,

Pasadena, California 91103

Received 13 December 1976; accepted 10 February 1977

1 Hewish, A., Scott, P. F. \& Wills, D. Nature 203, 1214-1217 (1964). ${ }^{2}$ Hewish, A., Dennison, P. A. \& Pilkington, J. D. H. Nature 209, 1188 - 1189 (1966). Dennison, P. A. \& Hewish, A. Nature 213, 343-346 (1967).

Ekers, R. D. \& Little, L. T. Astr. Astrophys. 10, 310-316 (1971)

Watanabe, T. Shibasaki, K. \& Kakinuma, T.J. geophys. Res. 79, 3841-3843 Watanabe,

Coles, W. A., Rickett, B. J. \& Rumsey, V. H. in Solar Wind Three (ed. Russell, C. T.) 351-367 (University of California, Los Angeles, 1974)

9 Hundhausen, A. J. Coronal Expansion and Solar Wind (Springer-Verlag, New York, 1972).

Barnes, A. Rev. Geophys. Space Phys. 13, 1049-1053 (1975)

11 Woo, R., Yang, F. C., Yip. K. W. \& Kendall, W. B. Astrophys, J. 208 (1976).

12 Woo, R., Yang, F. C.' \& Ishimaru, A Astrophys. 208 (1976).

13 Woo, R. Astrophys. J. 201, 238-248 (1975).

\section{Tidal acceleration of the moon deduced from observations of artificial satellites}

Douglas et al. ${ }^{1}$ demonstrated the existence of an apparent latitude dependence of tidal friction by determining disparate values of the second degree Love number $\left(k_{2}\right)$ from perturbations of the inclinations of the GEOS- 1 and GEOS- 2 satellites. Lambeck et $a l .^{2}$ correctly explained this phenomenon as being due to neglect of ocean tide perturbations. Parameter values for some ocean tide components have been obtained from several satellites ${ }^{3}$, but parameter values for the $M_{2}$ tide, the dominant $(85 \%)$ effect of the oceans on the tidal acceleration of the Moon, have not been published. Using an improved method for computing mean elements, we $\mathrm{e}^{4}$ obtained an observation equation for the $M_{2}$ tide from the satellite 1967-92A. Applying this technique to the satellite GEOS-3, we now obtain an additional observation equation for the $M_{2}$ tide. As shown in ref. 2, solid and fluid tide effects on satellites cannot be separated, requiring assumption of the solid tide amplitude and phase parameters for a fluid tide solution. Assuming $k_{2} \cdots 0.30, \delta_{2}=0$, and using the values of Lambeck for the minor $O_{1}$ and $N_{22}$ contributions to $\dot{h}_{1}$, our fluid tide parameters for the $M_{2}$ ocean tide yield the value of the tidal acceleration $\dot{n}_{\mathrm{t}}=-27.4 \pm 3$ arc $\mathrm{s}(100 \mathrm{yr})^{-2}$, in excellent agreement with the value $\dot{n}_{\mathrm{t}}=-27.2 \pm 1.7$ arc s $(100 \mathrm{yr})^{-2}$ obtained by Muller $^{6}$ from a combination of ancient and modern observations. These two values are lower than the value $\dot{n}_{\mathrm{t}}=-35 \pm 4$ arc s $(100 \mathrm{yr})^{-2}$ obtained from numerical ocean tide models ${ }^{5}$. Our assumption of a negligible solid tide phase angle is supported by a recent determination by J. T. Kuo (personal communication) that the phase angle obtained from a transcontinental network of tidal gravimetric stations is $<1^{\circ}$. Changes $\leqslant 0.5^{\circ}$ in solid tide phase angle change our result for the combined solid/fluid $\dot{n}_{\mathrm{t}}$ by no more than 1 arc $\mathrm{s}$ per (100yr) (ref. 2).

\section{C. GOAD}

B. C. Douglas

National Oceanic and Atmospheric Administration,

National Ocean Survey,

Geodetic Research and Development Laboratory,

Rockville, Maryland 20852

Received 18 January; accepted 16 February 1977.

${ }_{1}$ Douglas, B. C.., Klosko, S. M., Marsh, J. C. \& Williamson, R. G. Celestial Mechanics 10, 165-168 (1974).

12,421-434, (1974) 